\title{
Progressive Deforming Meshes based on Deformation Oriented Decimation
}

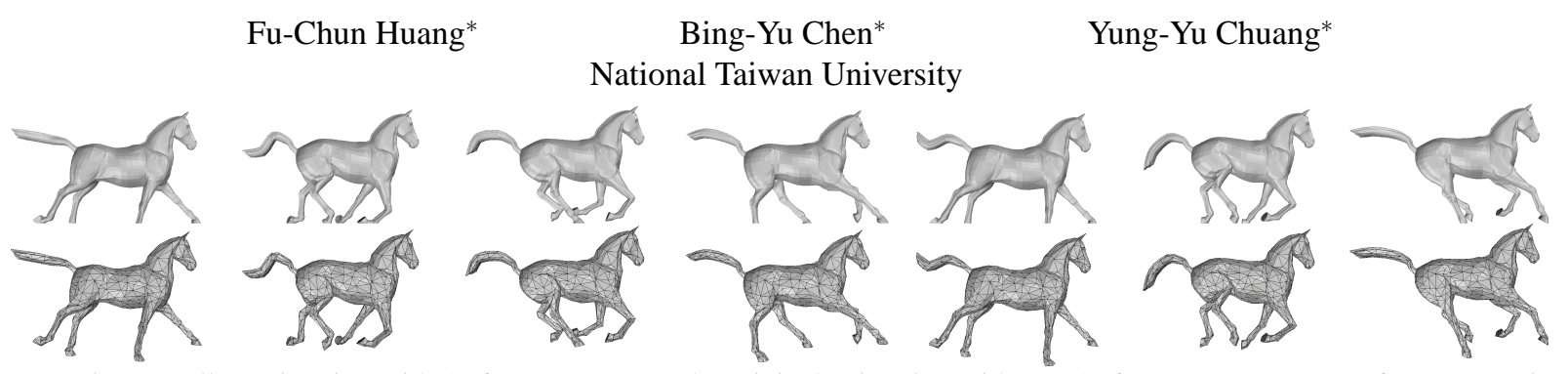

Figure 1: A horse-gallop animation with 48 frames. Upper: The original animation with 16,843 faces. Bottom: 1,588 faces approximation.

\section{Introduction}

Progressive Meshes (PM) presented by Hoppe [1996] is a method for representing a static 3D model with continuous levels-of-details (LODs) based on iterative edge-collapse/vertex-split operations. Besides static models, animation models or so-called deforming meshes are more widely used recently. Some previous methods simplify the deforming meshes primarily only based on the geometric features, however, in this poster we take the temporal and geometric features together into consideration.

\section{Deformation Oriented Decimation}

Our deformation oriented decimation (DOD) algorithm is based on Garland and Heckbert's QSlim algorithm [1997]. To measure the contraction cost for an edge, QSlim utilizes the quadratic error metric (QEM), which measures the total squared distance of a vertex to the two sets of planes. The area-weighted QEM error function $\Delta_{i j}$ for a vertex $\mathbf{v}$ to replace the edge $\left(\mathbf{v}_{i}, \mathbf{v}_{j}\right)$ is

$$
\Delta_{i j}(\mathbf{v})=w_{i} \sum_{\mathbf{p} \in \mathbf{P}\left(\mathbf{v}_{i}\right)} \mathbf{v}^{T}\left(\mathbf{p p}^{T}\right) \mathbf{v}+w_{j} \sum_{\mathbf{p} \in \mathbf{P}\left(\mathbf{v}_{j}\right)} \mathbf{v}^{T}\left(\mathbf{p} \mathbf{p}^{T}\right) \mathbf{v}=\mathbf{v}^{T} \mathbf{Q}_{i j} \mathbf{v},
$$

where $w_{i}=A_{i}$ is the total area of triangles adjacent to $\mathbf{v}_{i}, w_{j}$ is defined similarly, and $\mathbf{P}()$ denotes a set of planes. Hence, the QEM cost QEM ${ }_{i j}$ for contracting an edge $\left(\mathbf{v}_{i}, \mathbf{v}_{j}\right)$ is defined as $\Delta_{i j}\left(\mathbf{u}_{i j}\right)$, in which $\mathbf{u}_{i j}$ is the vertex minimizing $\left.\Delta_{i j}(\mathbf{v})\right)$.

Mohr and Gleicher [2003] extended the simplification for static model to deforming meshes to provide a deforming sensitive decimation (DSD) method. The DSD contraction cost for edge $\left(\mathbf{v}_{i}, \mathbf{v}_{j}\right)$ is defined as

$$
\operatorname{DSD}_{i j}=\sum_{t=1}^{f} \mathrm{QEM}_{i j}^{t}=\sum_{t=1}^{f} \mathbf{u}_{i j}^{t}{ }^{T} \mathbf{Q}_{i j}^{t} \mathbf{u}_{i j}^{t},
$$

where $\mathbf{u}_{i j}^{t}$ minimizes the QEM cost for the edge $\left(\mathbf{v}_{i}, \mathbf{v}_{j}\right)$ at frame $t$. Hence, DSD tends to preserve edges that belong to geometric features more often.

The DSD algorithm sums the QEM error metrics and is only concerned with the geometric features in each static model. This errormodel ignores the features occurred during the deformation in the temporal domain. Hence, we propose a DOD metric which incorporates a deformation cost into the DSD cost. The deformation $\operatorname{cost} \xi_{i j}$ is defined to measure the deformation by its average edge length change. The average length change for an edge $\left(\mathbf{v}_{i}, \mathbf{v}_{j}\right)$ can be defined as $\overline{\Delta l}_{i j}=\sum_{t=1}^{f-1} \Delta l_{i j}^{t}$, where $\Delta l_{i j}^{t}=\left|l_{i j}^{t+1}-l_{i j}^{t}\right|$ is the edge length change from frame $t$ to $t+1$ and $l_{i j}^{t}$ is the edge length of the edge $\left(\mathbf{v}_{i}, \mathbf{v}_{j}\right)$ at frame $t$. An edge with larger $\overline{\Delta l}_{i j}$ means that, on average, this edge performs more deformation. If we contract it, the

*e-mail: \{jonash,robin,cyy $\} @$ cmlab.csie.ntu.edu.tw

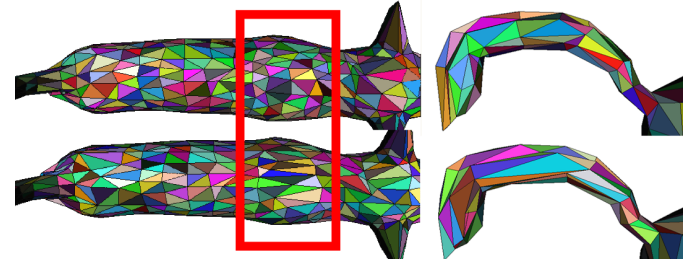

Figure 2: Upper: DOD result; Bottom: DSD result.

deformation performed by this edge will be lost. Hence, we should prefer to contract edges with smaller $\overline{\Delta l}_{i j}$ first.

Similar to area-weighted QEM, we would like to assign higher weights to edges of the triangles with larger areas. Hence, we use the average area $\bar{A}_{i j}=\sum_{t=1}^{f} A_{i j}^{t}$ as the weight, where $A_{i j}^{t}$ is the sum of areas of the two triangles sharing edge $\left(\mathbf{v}_{i}, \mathbf{v}_{j}\right)$. Thus, we define the deformation cost $\xi_{i j}$ for an edge $\left(\mathbf{v}_{i}, \mathbf{v}_{j}\right)$ and the DOD cost as:

$$
\xi_{i j}=w_{i j} * \bar{A}_{i j} *\left(\overline{\Delta l}_{i j}\right)^{2} \text {, and } \mathrm{DOD}_{i j}=\mathrm{DSD}_{i j}+\xi_{i j},
$$

where $w_{i j}$ is another weight defined as $w_{i j}=\frac{\max _{t} \Delta l_{i j}^{t}-\overline{\Delta l}_{i j}}{\sqrt{\frac{1}{f-1} \sum_{t=1}^{f-1}\left(\Delta t_{i j}^{t}-\overline{\Delta l}_{i j}\right)^{2}}}$. The denominator of the above equation is the standard deviation of length changes. This weight represents the normalized maximum deformation and helps to preserve the area with sudden large deformation.

\section{Result}

The result of our method is shown in Figure 2 (Upper) which shows two simplified deforming meshes. The left is a dog-walking animation which has a lot of motion around the neck; the right is a horsegallop animation whose tail swings up and down constantly. For the both animation, the DSD method preserves excessive polygons on the face portion while takes less care on the deforming regions, but our method successfully preserves both more polygons and better triangulation for such deforming regions. Our results indicate that even regions are not geometric features across every frame, the deformation cost defined previously can handle the deforming aspect.

\section{References}

Garland, M., and Heckbert, P. S. 1997. Surface simplification using quadric error metrics. In Proceedings of SIGGRAPH 97, 209-216.

Hoppe, H. 1996. Progressive meshes. In Proceedings of SIGGRAPH 96, 99-108.

Mohr, A., And Gleicher, M. 2003. Deformation sensitive decimation. Tech. rep., University of Wisconsin. 